\title{
Hiwi downregulation, mediated by shRNA, reduces the proliferation and migration of human hepatocellular carcinoma cells
}

\author{
YINGJUN XIE，YONGSHENG YANG，DEGANG JI，DAN ZHANG，XIAOXIAO YAO and XUEWEN ZHANG \\ Department of Hepatobiliary-Pancreatic Surgery, China-Japan Union Hospital of Jilin University, \\ Changchun, Jilin 130033, P.R. China
}

Received April 1, 2014; Accepted July 28, 2014

DOI: $10.3892 / \mathrm{mmr} .2014 .2847$

\begin{abstract}
The Piwi subfamily is one of two Argonaute family proteins, which are characterized by the presence of Piwi and Piwi-Argonaute-Zwille domains, and are well known for their role in RNA silencing. Hiwi, a human member of the Piwi subfamily, is restricted to the germ line, where it binds Piwi-interacting RNAs and functions in stem cell self-renewal and gametogenesis. Previous reports have indicated that abnormal Hiwi expression may be associated with a poor prognosis of numerous types of human cancer, including hepatocellular carcinoma (HCC). However, little is currently known about the oncogenic role of Hiwi in HCC. In the present study, it was confirmed that Hiwi is overexpressed at both the mRNA and protein level, in HCC specimens, as well as in MHCC97L and MHCC97H HCC cell lines. A lentivirus-mediated small hairpin RNA (shRNA) targeting Hiwi was constructed and used to infect MHCC97L and MHCC $97 \mathrm{H}$ cells. Relative Hiwi mRNA and protein expression levels were determined by quantitative polymerase chain reaction and western blot analysis, respectively. Cell proliferation, migration and invasion were determined using cell count, scratch and Transwell assays, respectively. Hiwi mRNA and protein expression was significantly downregulated in HCC cells in response to transduction with the lentivirus-mediated shRNA. Furthermore, the proliferative, migrative and invasive properties of the shRNA-transduced cells were significantly decreased. Therefore, Hiwi downregulation mediated by shRNA, may reduce the proliferation and migration of HCC cells. These results indicate that Hiwi may have an important
\end{abstract}

Correspondence to: $\mathrm{Dr}$ Xuewen Zhang, Department of Hepatobiliary-Pancreatic Surgery, China-Japan Union Hospital of Jilin University, 126 Xiantai Street, Changchun, Jilin 130033, P.R. China

E-mail: xuewen_zhang926@163.com

Key words: Hiwi, shRNA, proliferation, migration, human hepatocellular carcinoma cells role in the progression of HCC and may be a target for anticancer therapy.

\section{Introduction}

The Argonaute (Ago) proteins are defined by the presence of Piwi and Piwi-Argnaute-Zwille domains (1), which are expressed in both prokaryotic and eukaryotic organisms. In eukaryotes, Ago proteins are most well known for their role in RNA silencing (2). In mammals there are eight genes $(3,4)$, which encode the Ago and Piwi subfamilies $(5,6)$. The human Piwi subfamily is comprised of Hiwi1, Hiwi2, Hiwi3 and HILI, which are encoded for by genes on chromosomes 12, 11, 22 and 8, respectively (7). Ago proteins, which are ubiquitously expressed in numerous organisms, whereas in the majority of organisms investigated so far, including humans, the expression of Piwi proteins is restricted to the germ line, where they bind Piwi-interacting RNAs (piRNAs) $(2,5,6,8)$. piRNAs are a class of small non-coding RNAs (ncRNAs), which are involved in gene expression regulation. ncRNAs have been previously identified in almost all eukaryotic species, including humans $(4,9,10)$. In order to perform their effector functions, small ncRNAs must be incorporated into Ago complexes, resulting in the formation of highly specialized small-RNA-binding molecules which function in RNA-silencing pathways.

The Piwi gene family is highly conserved and exerts an essential role in stem cell self-renewal, gametogenesis, and RNA interference (RNAi) in diverse organisms (11). Hiwi is involved in human germ cell proliferation and maintenance, and the overexpression of the molecule has been indicated as a cause of malignant testicular germ cell tumors (11-13). The abnormal expression of Hiwi has also been suggested to be associated with a poor prognosis in numerous types of cancer, such as human pancreatic adenocarcinoma (14), human esophageal squamous cell carcinoma (15), colorectal cancer (16) and human gastric cancer (17). The alterations to Hiwi mRNA expression has been previously reported to increase the risk of tumour-related mortality in male patients with pancreatic adenocarcinoma (14). The cytoplasmic expression of Hiwi in esophageal cancer cells is significantly associated with a higher histological grade, clinical stage, 
and a poorer clinical outcome (15). PiwiL2 expression was shown to be upregulated and significantly correlated with a lower degree of differentiation, deep invasion and perineural invasion, in colorectal cancer (16). Furthermore, the overexpression of Hiwi in gastric cancer tissues was shown to be similar to that of $\mathrm{Ki}-67$, which is commonly used as a marker of proliferation, and the suppression of Hiwi inhibited the growth of gastric cancer cells and induced G2/M phase cell cycle arrest (17). These previous results suggest that Hiwi may be involved in the tumorigenesis of various cancers, and may be a target for anticancer therapy.

It has been observed by immunohistochemical analysis that Hiwi expression is significantly higher in human hepatocellular carcinoma (HCC) tissue, as compared with adjacent normal hepatic tissue (18). The overexpression of intratumoral Hiwi has been associated with a larger tumor size or intrahepatic metastasis, and was also shown to be an independent risk factor for overall and recurrence-free survival (19). These reports indicate that Hiwi may have a crucial role in the carcinogenesis of human HCC, and could serve as a potential biomarker or treatment target for HCC. In the present study, the overexpression of Hiwi was determined in HCC specimens, and in MHCC97L and MHCC97H HCC cell lines. In addition, a lentivirus-mediated small hairpin RNA (shRNA) targeting Hiwi was constructed and used to knockdown Hiwi expression, in order to investigate the influence of Hiwi on cancer cell proliferation and migration. It has been implied that Hiwi may have an important role in HCC progression, and it could be a potential target for anticancer therapy.

\section{Materials and methods}

HCC specimens, cell lines and culture conditions. A total of 60 intratumor and 48 peritumor specimens (used as a control; $>10 \mathrm{~mm}$ from the tumor edge) were resected from $\mathrm{HCC}$ patients. The patients were selected according to the pathological archives from the China-Japan Union Hospital, Jilin University (Jilin, China) between June 2006 and March 2010. All patients provided informed consent prior to the experiment. The fresh tissue specimens were immediately frozen in liquid nitrogen and stored at $-80^{\circ} \mathrm{C}$ post-resection, prior to being exposed to radiotherapy and chemotherapy. Clinical pathological data for each patient was available from the clinical records, and all of the information was assessed independently by three specialists. The present study was approved by the Medical Ethics Committee of China-Japan Union Hospital, Jilin University. MHCC97L, MHCC97H and HepG2 HCC cell lines, and the L02 normal hepatic cell line, were purchased from Shanghai Bioleaf Biotech Co., Ltd. (Shanghai, China). The cells were cultured at $37^{\circ} \mathrm{C}$ in a humidified atmosphere of $5 \% \mathrm{CO}_{2}$ in Dulbecco's Modified Eagle's medium (DMEM; Invitrogen Life Technologies, Carlsbad, CA, USA) supplemented with $10 \%$ fetal bovine serum (FBS; Gibco-BRL, Rockville, MD, USA).

RNA isolation and quantitative polymerase chain reaction ( $q P C R)$. Total RNA from the HCC cell lines and tissue specimens was extracted using the RNeasy ${ }^{\circledR}$ Plus Mini kit (Qiagen, Valencia, CA, USA), according to the manufacturer's instructions. qPCR (reverse transcription reaction, $42^{\circ} \mathrm{C}$ for $5 \mathrm{~min}$ and $95^{\circ} \mathrm{C}$ for $10 \mathrm{sec}$; PCR reaction, $95^{\circ} \mathrm{C}$ for $5 \mathrm{sec}$ and $60^{\circ} \mathrm{C}$ for $20 \mathrm{sec}$ for 40 cycles) was performed using a SYBR PrimeScript reverse transcription-qPCR kit (Takara Biotechnology Inc., Dalian, China) according to the manufacturer's instructions, and $\beta$-actin was used as an internal control. The primers for Hiwi and $\beta$-actin were synthesized by Sangon Biotech (Shanghai, China), according to previously reported sequences (19). The qPCR was performed with a Lightcycler 480 II (Roche Diagnostics GmbH, Mannheim, Germany). The data were normalized to $\beta$-actin and expressed as the fold change over control, and calculated using the $\Delta \Delta \mathrm{Ct}$ method (20).

Western blot analysis. HCC specimens for western blot analysis were homogenized prior to protein isolation. The homogenized HCC specimens and the HCC cultured cells were collected and lysed with ProteoJET ${ }^{\mathrm{TM}}$ Mammalian Cell Lysis reagent (Fermentas, Burlington, ON, Canada), according to the manufacturer's instructions. The resolved proteins were supplemented with a protease inhibitor cocktail (Complete Mini protease inhibitor cocktail; Roche Diagnostics $\mathrm{GmbH}$ ). The protein samples were then separated by SDS-PAGE (Sigma-Aldrich, St. Louis, MO, USA), and were transferred to polyvinylidene fluoride membranes (Pierce, Rockford, IL, USA), which were blocked in 5\% skimmed milk for $1 \mathrm{~h}$ at room temperature. The membranes were then incubated with either a 1:1,000 dilution Hiwi or a 1:3,000 dilution $\beta$-actin rabbit polyclonal antibody (Santa Cruz Biotechnology, Santa Cruz, CA, USA), at $4^{\circ} \mathrm{C}$ overnight. The membrane was then incubated with a peroxidase-conjugated secondary antibody $\left(1: 10^{6}\right.$; Sigma-Aldrich) for $1 \mathrm{~h}$ at room temperature. The blots were detected using the Enhanced Chemiluminescence Detection system (Amersham, Uppsala, Sweden), according to the manufacturer's instructions, and the Hiwi level was quantified via the relative gray value of the target band.

Lentivirus-mediated shRNA knockdown of Hiwi expression. The shRNAs targeting Hiwi (reference sequence, AF104260) were designed (9) by Ambion ${ }^{\circledR}$ (Life Technologies, Carlsbad, CA, USA), and a nonsense sequence was used as a control. The two shRNA-Hiwi sequences and one control sequence were confirmed using Basic Local Alignment Search Tool (BLAST) to a targeted sequence. The 9-nt hairpin sequence (TCAAGACG) was not homologous to Hiwi and was inserted between two shRNA-Hiwi sequences, TTTTTT, followed the antisense strand of shRNA, and acted as a termination sequence. BamHI and HindIII (Takara Biotechnology, Inc., Tokyo, Japan) were used as restriction endonucleases. cDNA oligonucleotides were synthesized and cloned into the lentivirus-based vector pGCSIL-GFP (GeneChem Co. Ltd., Shanghai, China). Lentiviruses were generated in HEK293T human embryonic kidney cells (American Type Culture Collection, Rockville, MD, USA) by co-transfection of each recombinant plasmid with a pHelper plasmid (GeneChem Co. Ltd.). HEK293T cells were cultured at $37^{\circ} \mathrm{C}$ in a humidified atmosphere of $5 \% \mathrm{CO}_{2}$ in DMEM with $10 \% \mathrm{FBS}$. The viral titer was quantitatively determined by counting the number of green fluorescent protein-positive cells post-viral infection, under a fluorescence microscope (Olympus, Tokyo, Japan). 
A

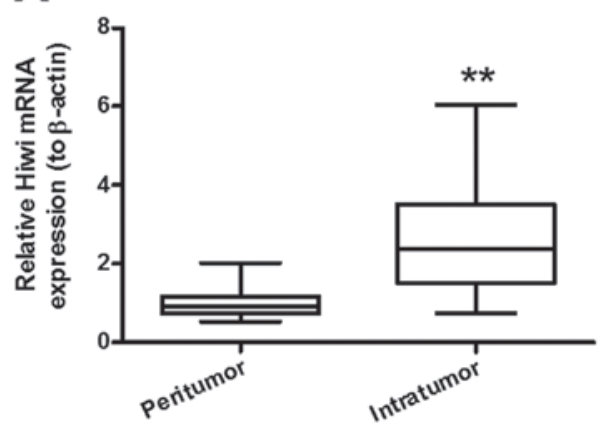

C

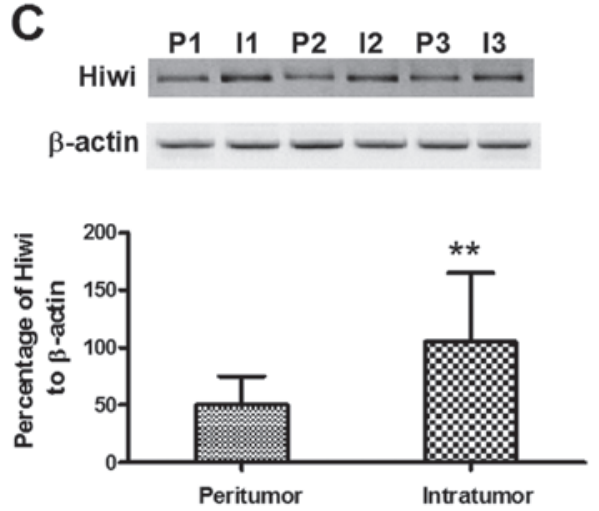

B

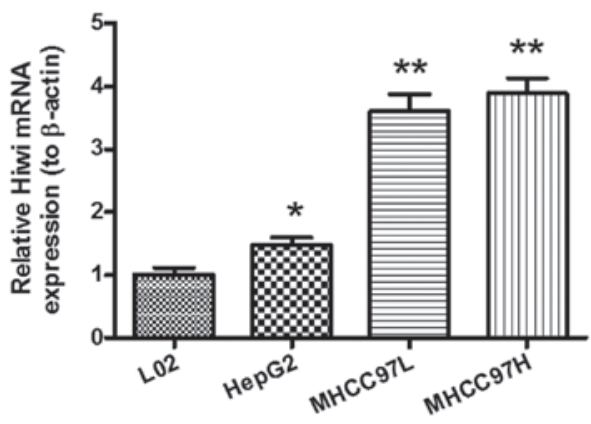

D

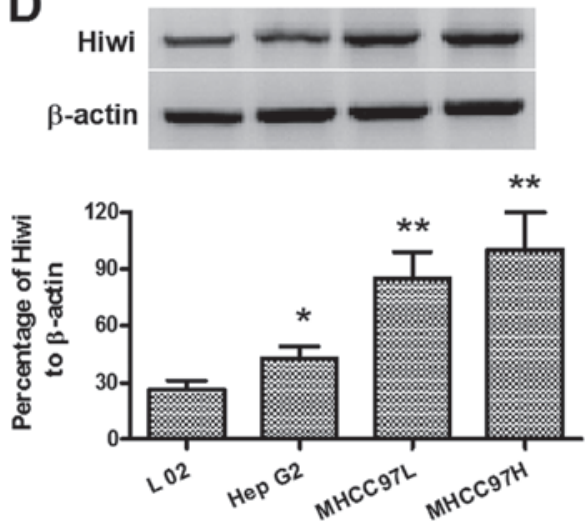

Figure 1. Overexpression of Hiwi in hepatocellular carcinoma specimens and cell lines. (A) Relative mRNA expression levels of Hiwi in the intratumor ( $\mathrm{n}=60$ ) and peritumor $(n=48)$ tissues of hepatocellular carcinoma (HCC), as determined by quantitative polymerase chain reaction. (B) Relative mRNA expression levels of Hiwi in HepG2, MHCC97L, and MHCC97H HCC cell lines, as compared with L02, normal hepatic cells. (C) Overexpression of relative Hiwi protein expression levels in the intratumor $(n=42)$ and peritumor $(n=27)$ tissues of HCC, as determined by western blot analysis. (D) Overexpression of relative Hiwi protein expression levels in HepG2, MHCC97L, and MHCC97H HCC cell lines, as compared with L02, normal hepatic cells, as determined by western blot analysis. The data represent the means \pm standard error of the mean. ${ }^{*} \mathrm{P}<0.05,{ }^{* *} \mathrm{P}<0.01$. I, intratumor; $\mathrm{P}$, peritumor.

For lentivirus transduction, MHCC97L and MHCC97H cells were subcultured at $1 \times 10^{4}$ cells/well in 12-well culture plates. Once the cells had reached $70 \%$ confluence, they were transduced with the shRNA-harboring lentiviruses at a multiplicity of infection of 10 . The cells were propagated under G418 (1.5 mg/ml; Sigma-Aldrich) selection pressure.

Cell count and colony formation assays. A cell count assay was performed as described by previous methods (21). Briefly, the cells were plated in 12 -well plates and incubated at $37^{\circ} \mathrm{C}$ for different periods of time, the cells were then trypsinized and the number of viable cells was counted using a hemocytometer with trypan blue staining. A total of 100 cells were seeded into a 12-well plate. After 10 days, the cells were stained with $0.5 \%$ crystal violet (Sigma-Aldrich) in methanol for $10 \mathrm{~min}$. The colonies ( $>50 \mu \mathrm{m}$ in diameter) were counted directly on the plate.

Migration and invasion assay. For the scratch assay, the cells were cultivated to $85 \%$ confluence on 12 -well plates and then scratched with a $200 \mu 1$ pipette tip. The cellular growth was observed at 0 and $48 \mathrm{~h}$ post-scratch. For the invasion assay, Matrigel-coated Transwell migration chambers (Corning Costar, Cambridge, MA, USA) were used. The cells were seeded at a density of $5 \times 10^{4}$ cells/well, in serum-free media, in the upper chamber with the non-coated membrane $(8 \mu \mathrm{m}$ pore size; Millipore, Zug, Switzerland). The lower chamber contained media supplemented with $20 \%$ FBS, as a chemoattractant. The cells in the upper chamber were discarded, using cotton wool, following a $24 \mathrm{~h}$ incubation; the cells which had migrated into the lower chamber were counted using a light microscope.

Statistical analyses. All data are expressed as the means \pm standard error of the mean. The comparisons between two groups were conducted using a Student's t-test. Statistical analyses were performed using GraphPad Prism ${ }^{\circledR}$ software version 5.0 (GraphPad Software, La Jolla, CA, USA). A $\mathrm{P}<0.05$ was considered to indicate a statistically significant difference.

\section{Results}

Overexpression of Hiwi in hepatocellular carcinoma specimens and cell lines. In order to identify a possible pro-oncogenic role of Hiwi in HCC, the expression levels of Hiwi were determined in both the HCC specimens and cell lines. Relative Hiwi mRNA expression levels, determined by qPCR, were significantly upregulated in intratumor specimens $(n=60 ; 2.724 \pm 0.185)$, as compared with the peritumor specimens $(\mathrm{n}=48 ; 1.000 \pm 0.064)($ Fig. $1 \mathrm{~A})(\mathrm{P}<0.01)$. Relative Hiwi mRNA expression levels were also found to 
A

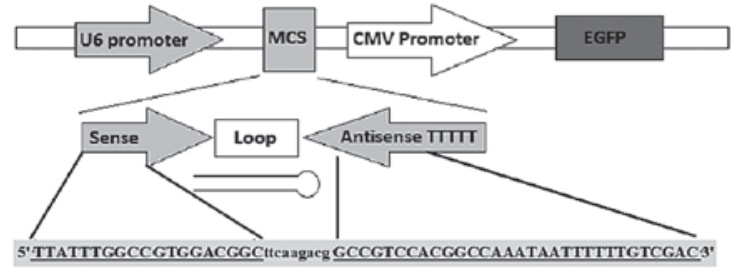

ShRNA-1 S'TIATTTGGCCGTGGACGGCHCaggag GCCGTCCACGCCCAAATAATTTTTTGTCGAC":

ShRNA-2 5' CGGTAATTGTGGTGAAGAAttcaagacgTTCTTCACCACAATTACCGTTTTTTGTCGAC 3

B

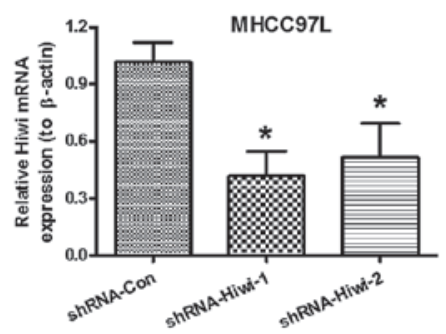

D

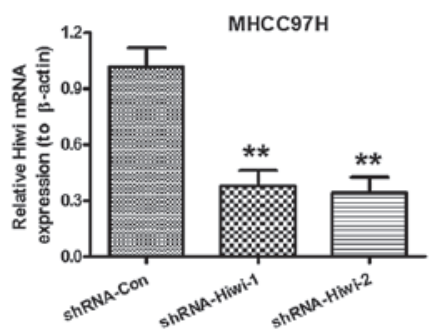

C

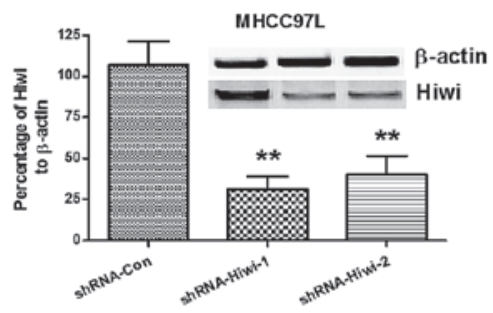

E

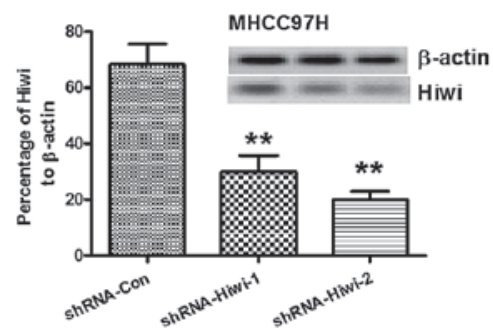

Figure 2. Suppression of Hiwi expression in MHCC97L and MHCC97H hepatocellular carcinoma (HCC) cell lines, mediated by lentivirus-mediated RNA interference. (A) Schematic diagram of the lentivirus vector, and structure of the cDNA template of the small hairpin RNA (shRNA) targeting Hiwi. The length of the palindrome sequence (19 or 20 nucleotide sense strand and antisense strand) was separated by a 9 nucleotide spacer, followed by four continuous thymines (T), as a termination signal. Two restriction enzymes (BamHI and XhoI) were added to the two termination ends. (B and C) Relative Hiwi mRNA expression levels in (B) MHCC97L or (C) MHCC97H HCC cells 24 h post-infection of lentivirus, expressing control or Hiwi shRNA, as determined by quantitative polymerase chain reaction. (D and E) Relative Hiwi protein expression levels in (C) MHCC97L or (E) MHCC97H HCC cells 48 h post-infection of lentivirus, expressing control or Hiwi shRNA, as determined by western blot analysis. $\beta$-actin was used as an internal control. The data represent the means \pm standard error of the mean of three independent experiments. ${ }^{*} \mathrm{P}<0.05,{ }^{* *} \mathrm{P}<0.01$, compared to the control group. CMV, cytomegalovirus; MCS, multiple cloning site; EGFP, enhanced green fluorescent protein.

be significantly upregulated in MHCC97L and $\mathrm{MHCC} 97 \mathrm{H}$ HCC cell lines, as well as HepG2, as compared with the L02 cells (Fig. 1B; $\mathrm{P}<0.01$ and $\mathrm{P}<0.05$, respectively). Relative Hiwi protein expression levels were determined by western blot analysis. Fig. 1C shows the upregulation of Hiwi protein expression in the intratumor specimens, as compared with the peritumor specimens $(\mathrm{P}<0.01)$. Figure $1 \mathrm{D}$ also indicates that there was a significant upregulation of Hiwi protein expression levels in MHCC97L, MHCC97H and HepG2 cells, as compared with L02 cells $(\mathrm{P}<0.01$ and $\mathrm{P}<0.05$, respectively). These results provide further evidence that Hiwi expression is upregulated in HCC tissue specimens and cell lines.

Hiwi knockdown in MHCC97L and MHCC97H HCC cell lines, by lentivirus-mediated RNAi. To further identify the pro-oncogenic role of Hiwi in HCC, Hiwi expression was knocked down by lentivirus-mediated shRNA. The recombinant lentiviruses (Lenti-Hiwi-shRNA-1 and Lenti-Hiwi-shRNA-2) and the control lentivirus (Lenti-shRNA-Con) were constructed with pRNAT-U6.1 vector, the cDNA sequence of Hiwi-shRNA-1, Hiwi-shRNA-2 or the control were inserted into the multiple cloning site, between the U6 and CMV promoters (Fig. 2A). The lentiviruses were packaged by transfecting the recombinant plasmid into HEK293T cells. The MHCC97L and MHCC97H cells were then transduced with the lentivirus (Lenti-Hiwi-shRNA-1, Lenti-Hiwi-shRNA-2, or Lenti-shRNA-Con), and the cells were selected for by G418 selection pressure, following three serial passages. The relative Hiwi mRNA and protein expression levels were determined in both cell lines, transduced with either shRNA-Hiwi or shRNA-Con, by qPCR and western blot analysis. As shown in Fig. $2 \mathrm{~B}$ and $\mathrm{C}$, the Hiwi mRNA expression levels in both cell lines were significantly reduced when transduced with either shRNA-Hiwi-1 or shRNA-Hiwi-2, as compared with shRNA-Con, post $24 \mathrm{~h}$ growth $(\mathrm{P}<0.05$ and $\mathrm{P}<0.01$, respectively). Western blot analysis also indicated reduced Hiwi protein expression levels in both cell lines post $48 \mathrm{~h}$ growth (Fig. 2D and E). These results indicated that lentivirus-mediated RNAi could efficiently and 
A

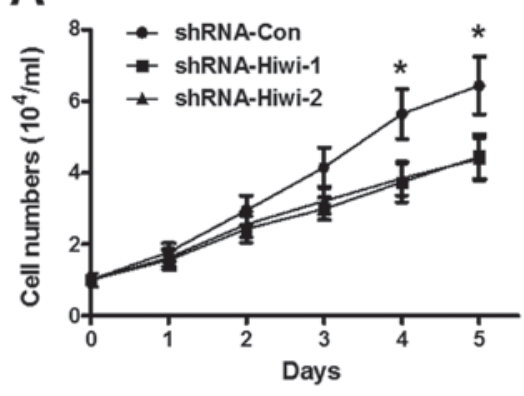

C

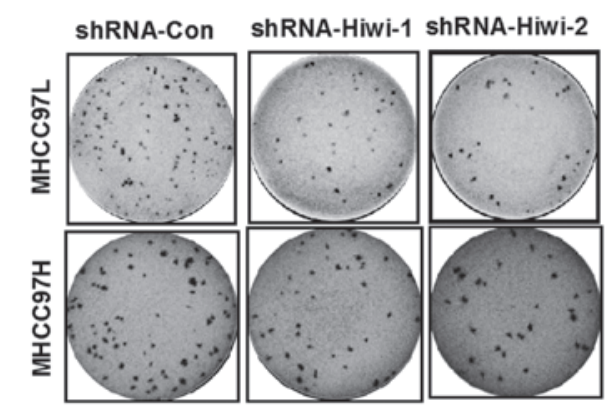

B

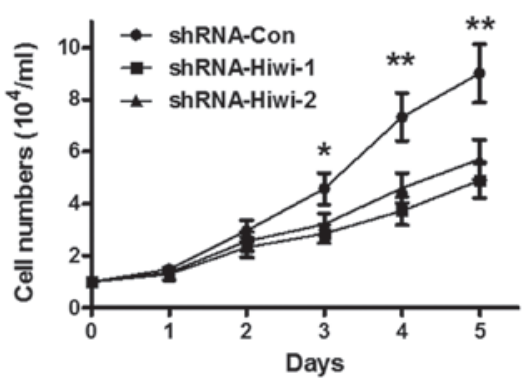

D

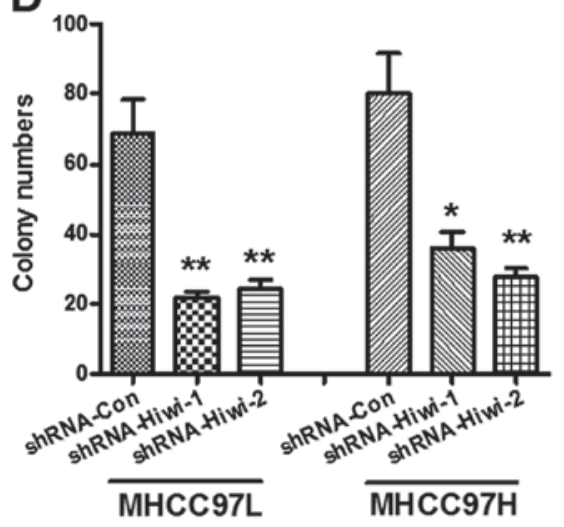

Figure 3. Hiwi silencing inhibits the proliferation of hepatocellular carcinoma (HCC) cells in vitro. (A and B) Significantly less cell proliferation was determined in the MHCC97L or MHCC97H HCC cells transduced with small hairpin RNA (shRNA)-Hiwi-1 or shRNA-Hiwi-2, as compared with the control shRNA group. The growth curve of both cells was evaluated by cell count assay. (C and D) Cell proliferation, by colony formation assay, in MHCC97L or MHCC97H cells with or without Hiwi knockdown by lentivirus-mediated RNA interference. The experiments were performed in triplicate. The data represent the means \pm standard error of the mean. ${ }^{*} \mathrm{P}<0.05,{ }^{* *} \mathrm{P}<0.01$.

specifically suppress Hiwi expression in both MHCC97L and MHCC97H HCC cells.

Hiwi knockdown inhibits HCC cell proliferation in vitro. The effects of Hiwi expression knockdown on the growth of HCC cells were assessed. The growth of HCC cells in vitro was measured by cell count and colony formation assays. The cell count assay was performed daily for five days. As shown in Fig. 3A, Hiwi silencing inhibited MHCC97L cell proliferation in a time-dependent manner. As compared with the control shRNA group, the cell numbers in the Hiwi-shRNA-1/2 groups ( $3.72 \pm 0.54 \times 10^{4}$ and $3.84 \pm 0.48 \times 10^{4}$, respectively) were significantly reduced four days post-inoculation as compared with the control group $\left(5.64 \pm 0.70 \times 10^{4}\right)(\mathrm{P}<0.05)$, and five days post-inoculation: $4.45 \pm 0.62 \times 10^{4}$ for shRNA-Hiwi-1 and $4.38 \pm 0.59 \times 10^{4}$ for shRNA-Hiwi-2, as compared with the control $\left(6.43 \pm 0.82 \times 10^{4}\right)(\mathrm{P}<0.05)$. The reduction of Hiwi protein expression levels was more significant in the MHCC $97 \mathrm{H}$ cells. Fig. 3B demonstrated that from three days post-inoculation, both Hiwi-shRNAs significantly inhibited HCC cell growth $(\mathrm{P}<0.05)$; the growth rate was more significantly reduced four and five days post-inoculation in response to both of the Hiwi-shRNAs $(\mathrm{P}<0.01)$. Furthermore, the colony forming abilities of both MHCC97L and MHCC97H cells transduced with either control shRNA or Hiwi-shRNA1/2 lentivirus, was evaluated. As shown in Fig. 3C and D, the colony numbers of MHCC97L or MHCC97H cells in the Hiwi-shRNA-1 and Hiwi-shRNA-2 groups were significantly reduced, as compared with the control shRNA group $(\mathrm{P}<0.01)$. These results provide further evidence of the role of Hiwi in the promotion of HCC cellular growth.

Hiwi knockdown decreases cell migration and invasion of $H C C$ cell growth in vitro. It is well known that cell migration is responsible for tumor metastasis (22). To further identify the pro-oncogenic function of Hiwi, the differences in the migration processes of $\mathrm{MHCC} 97 \mathrm{~L}$ and $\mathrm{MHCC} 97 \mathrm{H}$ cells transduced with either control shRNA or Hiwi-shRNA-1 or 2 was determined by scratch assay. The MHCC 97L or MHCC97H cells transduced with Hiwi-shRNA-1 or 2 exhibited significantly slower migration, as compared with the cells transduced with control shRNA (Fig. 4A and $\mathrm{B})(\mathrm{P}<0.01$ and $\mathrm{P}<0.05$, respectively). Furthermore, the invasive capabilities of the cells was determined using a Matrigel-coated Transwell assay, the invasive cell number was quantified in Fig. 4C. Consistent with the findings of the scratch assay, MHCC97L and MHCC97H cells transduced with Hiwi-shRNA1 or 2 had a significant reduction in cell invasive ability, as compared with the control shRNA-treated cells. These results indicated that Hiwi knockdown by shRNA may reduce the migration of HCC cells in vitro.

\section{Discussion}

$\mathrm{HCC}$ is one of the most common types of cancer and is characterized by high malignancy, particularly in Africa and 
A

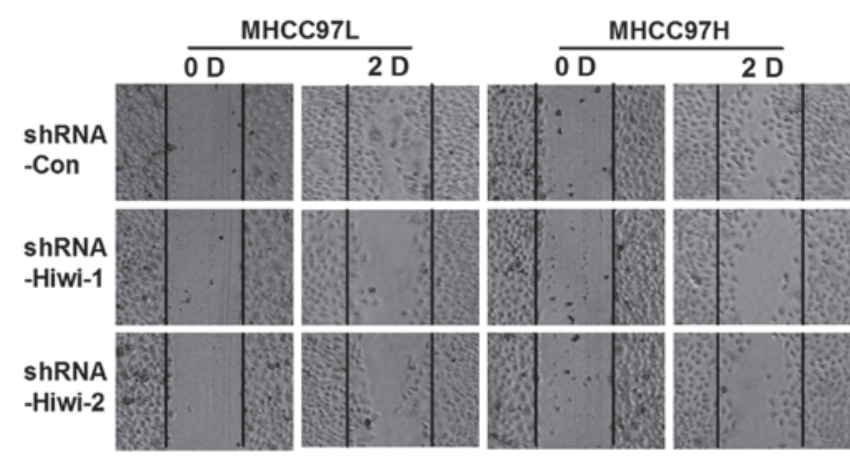

B

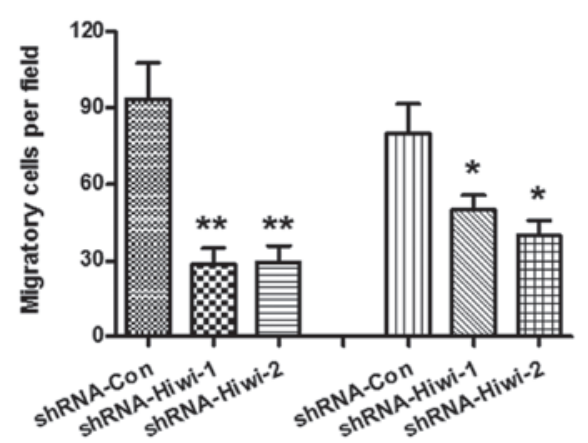

C

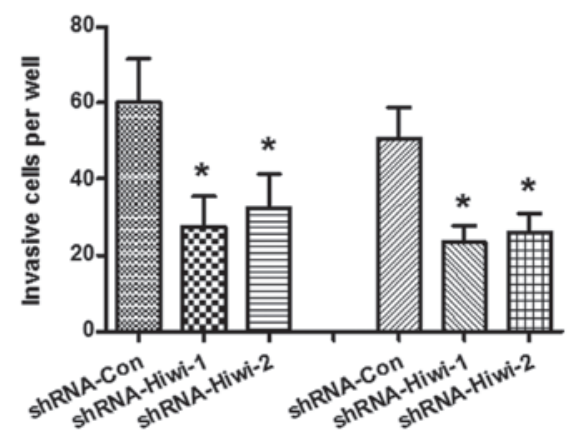

Figure 4. Hiwi silencing decreases cell migration and invasion of hepatocellular carcinoma (HCC) cells in vitro. (A) Migration of both MHCC97L and MHCC $97 \mathrm{H}$ HCC cells, after Hiwi expression was silenced by RNA interference, by cell scratch assay. Cell migration was observed 0 and 48 h post-scratch. Solid lines show the edges of the wound at the start of experiments. (B) The migration was calculated by the rate of cells filling the scratched area (left three columns, MHCC97L cells; right three columns, MHCC97H cells). (C) Cell invasion was determined by Matrigel-coated Transwell assay. The cells that crossed the Matrigel-coated filter were fixed, stained and counted (left three columns, MHCC97L cells; right three columns, MHCC97H cells). The data represent the means \pm standard error of the mean of three independent experiments. Statistical significance was shown as * $\mathrm{P}<0.05$, ${ }^{* *} \mathrm{p}<0.01$. $\mathrm{D}$, day; shRNA, small hairpin RNA. D, day; Con, control.

Asia, where it is the second most common cause of cancer mortality in China (23), with $\sim 600,000$ deaths each year worldwide (24). The predisposing factors for HCC include chronic infection with hepatitis $\mathrm{B}$ and $\mathrm{C}$ virus, alcohol abuse, and aflatoxin intake $(25,26)$, which all may induce cirrhosis, posing the highest risk of HCC development; $80 \%$ of HCCs develop from cirrhotic livers (27). The activation of oncogenes and inactivation of tumor suppressor genes have been identified as being associated with carcinogenesis and the progression of HCC. Numerous genes have been identified which are differentially expressed in HCC tumor tissue, as compared with paratumor tissue, which are oncogenic or tumor suppressive. These genes include IGF2, FAT10, SCARA5, DLK1, p53 and Zinc finger protein 267 (28-33). Previously, Hiwi has been indicated as being significantly overexpressed in human HCC tissue, as compared with adjacent normal hepatic tissue (18). Hiwi has also been shown to be an independent risk factor for overall survival and recurrence-free survival rates (19) in patients with HCC. These results imply that Hiwi may be an oncogenic regulator of human HCC.

In the present study, the overexpression of Hiwi in HCC specimens and cell lines was confirmed, at both the mRNA and protein expression levels. Both qPCR and western blot analysis revealed a significantly upregulated level of Hiwi expression in intratumor specimens, as compared with peritumor specimens. Hiwi overexpression was also identified in MHCC97L and MHCC97H HCC cell lines, as well as
HepG2, as compared with the L02 normal hepatic cells. To further identify the role of Hiwi in HCC, a lentivirus-mediated shRNA was used to knockdown Hiwi expression. The influence of the Hiwi knockdown was determined on the proliferation and migration of HCC cells. The results demonstrated that the Hiwi expression in both $\mathrm{MHCC} 97 \mathrm{~L}$ and $\mathrm{MHCC} 97 \mathrm{H}$ cells was significantly reduced following Hiwi-specific shRNA transduction, at both the mRNA and protein level. These results indicated that lentivirus-mediated shRNA may efficiently and specifically suppress Hiwi expression in both MHCC97L and MHCC97H cells.

The influence of the Hiwi knockdown on the proliferation and migration of HCC cells was evaluated. The cell count assay showed that cell proliferation was significantly reduced, in a time-dependent manner, in MHCC97L and $\mathrm{MHCC} 97 \mathrm{H}$ cells in the shRNA-Hiwi-1 or 2 groups, as compared with cells in the shRNA-Con group. The colony formation assay also indicated that the colony numbers of MHCC97L or MHCC97H cells in both the Hiwi-shRNA-1 and Hiwi-shRNA-2 groups were significantly lower, as compared with the shRNA-Con group. These results indicated that the shRNA-mediated Hiwi knockdown, resulted in growth inhibition of the HCC cells. The influence of Hiwi knockdown on the cell migration and invasion of HCC cells was determined by scratch and Transwell assays. The results demonstrated that the Hiwi knockdown in MHCC97L or MHCC97H cells, by Hiwi-shRNA-1 or 2, significantly reduced the migratory capacity of both cells. Hiwi-shRNA1 or 2 transduction also 
significantly reduced the invasiveness of MHCC97L and MHCC97H cells. These results indicated that Hiwi knockdown by shRNA reduced the migratory capacity of HCC cells in vitro.

In conclusion, the present study confirmed the overexpression of Hiwi in HCC specimens and cell lines, at both the mRNA and protein expression levels. It was also identified that Hiwi expression knockdown, in MHCC97L and MHCC97H HCC cell lines, by lentivirus-mediated RNAi resulted in inhibition of cellular growth, migration and invasion in vitro. The results of the present study implied that Hiwi has an oncogenic role in HCC.

\section{References}

1. Bohmert K, Camus I, Bellini C, Bouchez D, Caboche M and Benning C: AGO1 defines a novel locus of Arabidopsis controlling leaf development. EMBO J 17: 170-180, 1998.

2. Hall TM: Structure and function of argonaute proteins. Structure 13: 1403-1408, 2005.

3. Carmell MA, Xuan Z, Zhang MQ and Hannon GJ: The Argonaute family: tentacles that reach into RNAi, developmental control, stem cell maintenance, and tumorigenesis. Genes Dev 16: 2733-2742, 2002.

4. Meister G and Tuschl T: Mechanisms of gene silencing by double-stranded RNA. Nature 431: 343-349, 2004.

5. Hutvagner G and Simard MJ: Argonaute proteins: key players in RNA silencing. Nat Rev Mol Cell Biol 9: 22-32, 2008.

6. Peters L and Meister G: Argonaute proteins: mediators of RNA silencing. Mol Cell 26: 611-623, 2007.

7. Höck J and Meister G: The Argonaute protein family. Genome Biol 9: 210, 2008.

8. Houwing S, Kamminga LM, Berezikov E, et al: A role for Piwi and piRNAs in germ cell maintenance and transposon silencing in Zebrafish. Cell 129: 69-82, 2007.

9. Elbashir SM, Harborth J, Lendeckel W, Yalcin A, Weber K and Tuschl T: Duplexes of 21-nucleotide RNAs mediate RNA interference in cultured mammalian cells. Nature 411: 494-498, 2001.

10. Sana J, Faltejskova P, Svoboda M and Slaby O: Novel classes of non-coding RNAs and cancer. J Transl Med 10: 103, 2012.

11. Qiao D, Zeeman AM, Deng W, Looijenga LH and Lin H: Molecular characterization of hiwi, a human member of the piwi gene family whose overexpression is correlated to seminomas. Oncogene 21: 3988-3999, 2002.

12. Skotheim RI, Kraggerud SM, Fossa SD, et al: Familial/bilatera and sporadic testicular germ cell tumors show frequent genetic changes at loci with suggestive linkage evidence. Neoplasia 3: 196-203, 2001

13. Summersgill B, Osin P, Lu YJ, Huddart R and Shipley J: Chromosomal imbalances associated with carcinoma in situ and associated testicular germ cell tumours of adolescents and adults. Br J Cancer 85: 213-220, 2001.
14. Grochola LF, Greither T, Taubert H, et al: The stem cell-associated Hiwi gene in human adenocarcinoma of the pancreas: expression and risk of tumour-related death. Br J Cancer 99: 1083-1088, 2008.

15. He W, Wang Z, Wang Q, et al: Expression of HIWI in human esophageal squamous cell carcinoma is significantly associated with poorer prognosis. BMC Cancer 9: 426, 2009.

16. Oh SJ, Kim SM, Kim YO and Chang HK: Clinicopathologic implications of PIWIL2 expression in colorectal cancer. Korean J Pathol 46: 318-323, 2012.

17. Liu X, Sun Y, Guo J, et al: Expression of hiwi gene in human gastric cancer was associated with proliferation of cancer cells. Int J Cancer 118: 1922-1929, 2006.

18. Jiang J,Zhang H, Tang Q, Hao B and Shi R: Expression of HIWI in human hepatocellular carcinoma. Cell Biochem Biophys 61: 53-58, 2011.

19. Zhao YM, Zhou JM, Wang LR, et al: HIWI is associated with prognosis in patients with hepatocellular carcinoma after curative resection. Cancer 118: 2708-2717, 2012

20. Livak KJ and Schmittgen TD: Analysis of relative gene expression data using real-time quantitative PCR and the 2(-Delta Delta C(T)) Method. Methods 25: 402-408, 2001.

21. Li Z, Tian T, Lv F, et al: Six1 promotes proliferation of pancreatic cancer cells via upregulation of cyclin D1 expression. PLoS One 8: e59203, 2013

22. Parkin DM, Bray F, Ferlay J and Pisani P: Global cancer statistics, 2002. CA Cancer J Clin 55: 74-108, 2005.

23. Xu C, Liu S, Fu H, et al: MicroRNA-193b regulates proliferation, migration and invasion in human hepatocellular carcinoma cells. Eur J Cancer 46: 2828-2836, 2010.

24. Aravalli RN, Steer CJ and Cressman EN: Molecular mechanisms of hepatocellular carcinoma. Hepatology 48: 2047-2063, 2008.

25. Schafer DF and Sorrell MF: Hepatocellular carcinoma. Lancet 353 : 1253-1257, 1999.

26. Thorgeirsson SS and Grisham JW: Molecular pathogenesis of human hepatocellular carcinoma. Nat Genet 31: 339-346, 2002.

27. Llovet JM, Burroughs A and Bruix J: Hepatocellular carcinoma. Lancet 362: 1907-1917, 2003.

28. Iizuka N, Oka M, Yamada-Okabe $\mathrm{H}$, et al: Comparison of gene expression profiles between hepatitis B virus- and hepatitis C virus-infected hepatocellular carcinoma by oligonucleotide microarray data on the basis of a supervised learning method. Cancer Res 62: 3939-3944, 2002.

29. Oliva J, Bardag-Gorce F, French BA, et al: Fat10 is an epigenetic marker for liver preneoplasia in a drug-primed mouse model of tumorigenesis. Exp Mol Pathol 84: 102-112, 2008.

30. Huang J, Zheng DL, Qin FS, et al: Genetic and epigenetic silencing of SCARA5 may contribute to human hepatocellular carcinoma by activating FAK signaling. J Clin Invest 120: 223-241, 2010.

31. Huang J, Zhang X, Zhang M, et al: Up-regulation of DLK1 as an imprinted gene could contribute to human hepatocellular carcinoma. Carcinogenesis 28: 1094-1103, 2007.

32. Okada T, Iizuka N, Yamada-Okabe H, et al: Gene expression profile linked to p53 status in hepatitis $\mathrm{C}$ virus-related hepatocellular carcinoma. FEBS Lett 555: 583-590, 2003.

33. Schnabl B, Valletta D, Kirovski G and Hellerbrand C: Zinc finger protein 267 is up-regulated in hepatocellular carcinoma and promotes tumor cell proliferation and migration. Exp Mol Pathol 91: 695-701, 2011. 